\title{
Assessment of Knowledge, Attitude and Practice toward Menstruation among Adolescent Girls at Sohag City
}

\author{
Manal Mohamad Abed, Youssria Elsayed Yousef \& Neama Mohamad El-Maghraby.
}

Lecturer o f, Department of Pediatric Nursing, Faculty of Nursing, Sohag University Egypt.

Assistant Professor, Department of Pediatric Nursing, Faculty of Nursing, Sohag University Department of Community Health Nursing, Faculty of Nursing, Assiut University Egypt.

\begin{abstract}
Aim of the study: To assess the knowledge, attitude and practice toward menstruation among adolescent girls at Sohag City Methods: A descriptive cross-sectional design was utilized for the study. A convenient sample of 100 adolescent girls was recruited for the study. A self-administrated questionnaire and Menstruation Attitude Questionnaire (MAQ) were used for data collection. Results: The level of knowledge was more significantly higher among urban girls and those with a higher level of education. Two-thirds had a satisfactory level of knowledge; more than two-thirds used sanitary pads as the ideal use during menstruation. The participants agreed that menstruation is a debilitating among 42 girls (42\%), a bothersome among 15 girls (15\%) and natural event among 53 girls (53\%). Furthermore, they agreed that they can anticipate their menstruation among 80 girls $(80 \%)$ and they denied the effect of it among 8 girls (8\%). The majority of participants were informed about menstruation from their mothers as the first source of information. There was a positive correlation between participants' score of knowledge and their attitude toward menstruation. Conclusions and Recommendations: adolescent girls had a satisfactory level of knowledge about menstruation. Their mothers were the main source of information. The level of knowledge positively correlated with the girls attitude toward menstruation It is recommended to prepare girls for menstruation before menarche through providing them with well-planned school health program for girls and mothers about menstruation by well-trained educators.
\end{abstract}

Keywords: Knowledge, Attitude, Practice, Menstruation \& Adolescent Girls.

\section{Introduction}

Menstruation is a natural phenomenon experienced by almost all girls that occur throughout the reproductive years. It is influenced by physiological and pathological events occurring throughout the life time of the girl. Meanwhile, it is still poorly understood and considered a taboo that prevents individuals to speak about it (Nese, 2013).

All the current studies indicated that menstruation has little or no effect on performance (Çakmakci et al., 2005). Environment culture and religion play important roles on attitudes toward menstruation. Regarding religious restrictions during menstruation, a menstruating Muslim girl is not allowed to enter the mosque for praying, touch the Qur'an, or fast in Ramadan (Sadiq \& Salih, 2013)

Different studies indicated that perceptions and severity of psychological and physical symptoms during menstruation are mostly affected by negative or positive attitudes toward menstruation (Marván et al., 2005). Menstruation-related information of people determines their response to this event. Some studies have shown that negative physical and psychological changes during menstruation period are associated with the perception of menstruation (Houston et al., 2006).
Girls may be unfamiliar with what is normal and may not inform their parents about menstrual irregularities or missed menses (Diaz et al., 2006). The onset of menstruation in adolescence is a phenomenon that signals reproductive maturity and should not be seen as an abnormal condition or disease. Adolescent girls often do not receive accurate information about menstrual health because of culturally specific practices that lead to incorrect and unhealthy behaviors (Moloud et al., 2012).

In recent years, reproductive health care has been a primary concern and a program about reproductive health care has been started. Education regarding menstrual health has changed and conducting health promotion projects focused on puberty (Nese, 2013). While menstruation has been discussed broadly, negative and positive cultural elements about menstruation are still effective on attitudes towards menstruation. Menstruation has important implications on the physical and emotional wellbeing of adolescents' reproductive health. The way girls perceive menstruation has an effect on their own body image, gender identity, self-acceptance, sexual and health behavior (Çevirme et al., 2010). Most of the girls believe that menstruation affects their performance negatively and recently many girls and 
women refuse to join physical activities during menstruation (Nese, 2013).

Most girls experience some degree of pain and discomfort in their menstruation period, which could have important impacts on their daily activities, and disturb their productivity at home or at their work place. In these instances, they should consult a physician to relieve their pain and other relevant symptoms of dysmenorrheal (Wong \& Khoo, 2011). According to many reports, the prevalence of dysmenorrhea is very high, and at least $50 \%$ of girls experience this problem throughout their reproductive years (Sudenshana and Dasgupta, 2012). This problem not only causes fear in approximately one-fifth of the female population, but also causes many social, physical, psychological, and economic problems for them around the world. It has been also shown that dysmenorrhea is considered as the main cause of absence from the school among young girl students (LiPing \& Ming, 2011).

Menstrual hygiene is the personal hygiene during menstruation. It includes bathing daily for comfort, using clean, dry absorbent material and disposal of used pads/material in clean environmentally acceptable, safe methods and to feel fresh, keep perineal area clean from anterior to posterior(Eswi et al., 2012). Menstrual hygiene, a very important risk factor for reproductive tract infections, is a vital aspect of health education for adolescent girls.

Today millions of girls suffer from reproductive tract infections and their complications and they sometimes transmit it to their next generation. The girls who have a better understanding of this natural phenomenon and using safe healthy practices are definitely less vulnerable, so that health education by teachers, parents, and the media is important in addressing misconceptions about menstrual health (Shokry et al., 2012).

Adinma (2008), reported that faulty perceptions or misconception on menstruation and menstrual cycle will lead to faulty menstrual practices. In addition, Anjum et al., (2010), reported that attitudes toward menstruation may adversely affect women's body image, the perception of disease causation, diet, willingness to take medication, contraceptive use, and the ability to plan pregnancies.

Menstrual education is a vital aspect of health education. It is known that attitudes to menstruation and menstrual practices developed at menarche may persist throughout life (Jarrah and Kamel, 2012)

Adolescent females in schools may have their own attitude toward menstrual cycle. This attitude may be affected by cultural perspective, lack of knowledge, and embarrassment to speak about this normal phenomenon with their mothers at home or others. Also, there is scattered research that examines female attitude and knowledge and practice among adolescent girls in Egypt. Therefore, the current study may contribute to a better understanding of the Egyptian adolescent girls' attitude and knowledge and practice related to menstruation.

Before planning for health education programs about menstruation, a study of adolescents' knowledge of menstruation, and their beliefs and practices will help the health educator, maternity nurse, school nurse, community nurse discovers deficiencies in their knowledge and troubling misconception-related issues (Fetohy, 2007).

\section{Significance of the study}

In adolescents who experienced menstruation for the first time, menstrual hygiene management is constrained by practical, social, economic and cultural factors such as the expense of commercial sanitary pads, lack of water and latrine facilities, lack of private rooms for changing sanitary pads, and limited education about the facts of menstrual hygiene. Adolescents enter puberty unprepared and the information they receive is often selective and surrounded by taboos. In many curricula, there is an emphasis on the reproductive process but not on the practical issues.

The majority of girls usually have a lack of scientific knowledge and hygienic practice during menstruation and puberty. Also, girls often are reluctant to discuss this embracing topic with their care providers and often hesitate to seek help regarding the menstrual problem from external sources. So, girls should be educated about "menstruation and healthy menstrual cycle. UNICEF estimates that 1 in 10 school-age African girls do not attend school during menstruation. Similarly, World Bank statistics indicated that students have been absent from school 4 days every 4 weeks because of menstruation.

Addressing menstrual hygiene management directly contributes to improving maternal health. Due to its indirect effect on school absenteeism and gender discrepancy, poor menstrual hygiene and management may seriously hamper the realization of universal education and gender equality and women empowerment. However, menstrual hygiene management is an issue that is insufficiently acknowledged. Thus, the objective of the study is to assess the knowledge, attitudes and practice of adolescent girls. .

\section{Aim of the study}

Aim of the study is to assess knowledge, attitude and practice toward menstruation among adolescent girls at Sohag City 


\section{Research questions}

1-What is the knowledge about menstruation among adolescent girls?

2-What is the attitude of the adolescent girls toward menstruation?

3-What is the practice of the adolescent girls during menstruation?

4-Is there is a relationship between the menstrual knowledge and attitude among adolescent girls?

5-Is there is a relationship between the menstrual knowledge and practice among adolescent girls?

\section{Subjects and methods}

Research design: -A descriptive cross-sectional design was used.

\section{Subjects and settings}

The target population of this study consisted of all adolescent girls (12-18 years) from students enrolled in the preparatory and secondary schools at Sohag City. Two educational districts were selected that have combined preparatory and secondary schools where two of them were selected according to the selection of the authorities of Ministry of Education. A convenient sample of 100 adolescent girls were included who accepted to participate in this study (47 from one Omelmoemenen school and 53 from the Naser school).

Tools of data collection

- A self-administrated questionnaire was used as a tool for data collection; it included personal data related to age, residence, educational level, menstrual history and the student's knowledge about menstruation. The questionnaire included also variables on menstrual hygiene materials and knowledge about menstrual hygiene management such as the use of sanitary materials, ideal adsorbents, frequency at which sanitary pads should be changed,

- Questionnaire sheet: Menstrual Attitude Questionnaire (MAQ) was used as a data collection device in this study. MAQ is developed by (Firat et al., 2009) is the gold standard measurement of menstruation. The 33-item MAQ consisting of five subscales and items are scored on a Likert scale of strongly disagree to strongly agree. The original scale is scored per subscale and reported Cronbach's alpha coefficient of 0.95 to 0.972 . The validity and reliability of the questionnaire were tested by Kulakaç in Turkey.

- The scale was translated into Turkish and retranslated into English by specialists and retranslated into Arabic by the researchers of the current study because of the student's native language is originally Arabic. The test-retest reliability coefficients were found to be statistically significant $(\mathrm{p}=.000)$. The internal consistency reliability coefficient was 0.79 for the total MAQ. The questionnaire consists of 33 items and 5 factors, which consider menstruation as a debilitating event of 12 items, menstruation as a bothersome event of 6 items, menstruation as a natural event of 4 items, Anticipation and prediction of the onset of menstruation of 4 items and Denial of any effects of menstruation of 7 items. The converted MAQ is a Likert-type scale and has five rating scales of 1 strongly disagree to 5 strongly agree.

\section{Scoring system}

Scores were used to evaluate participant's knowledge about menstruation. Questions were scored as followed 1 mark for the correct answer and 0 marks for wrong or no answer. A total score of each aspect of $60 \%$ or more is considered satisfactory knowledge while less than $60 \%$ is unsatisfactory knowledge.

\section{Ethical considerations}

An approval was obtained from authorities of Ministry of Education and schools Objective of the study and its implication were explained to both schools administrators and the students. An oral consent was obtained from students who were willing to participate in the study and attained menarche. All information are confidential. Students were informed that their participation will be the involuntary basis and it will not affect their grades and evaluation.

\section{Field work}

A cross-sectional study was conducted from March to May 2015. Multistage sampling technique was used. Each school was first clustered into grades \& sections and then participants were selected by random method. The school authorities were contacted and informed about the nature and objective of the study. After obtaining the permission from the school authorities, the investigators visited the school as the pre-planned schedule for interviewing the adolescent girls. The purposes of the study were explained for adolescent girls and they were elective for participation and were assured regarding confidentiality.

The oral consent was taken from each participant. The data was collected by applying a 33 item questionnaire. The researcher with the help of one of the teachers has introduced herself to the students and the purpose of the visit and the way of the interview was explained to them. A direct interview was done by the researcher herself with each student separately and privately using a questionnaire composing of questions regarding knowledge attitude and practice during the menstrual cycle, which took around $20-25$ minutes. 
All questionnaires were distributed and collected on the same day trying to maintain the confidentiality of the collected data. A questionnaire with 33 items was designed by the authors and was initially tested on 10 adolescent girls with the similar characteristics of the main study subjects for construct and content validity. To develop the main questionnaire, the questionnaires items included the onset of menstruation (menarche), the source of information about menstruation and role of nutrition, exercise and physical activity, personal hygiene, use of medication, absenteeism from school and schoolgirls' attitude toward menstruation. The responses of the question were set in such a manner to be answered in the form of yes/no/true/false response.

\section{Pilot study}

Before the actual data collection, the questionnaire was pre-tested on $10 \%$ of the study subjects, with similar characteristics of the main study subjects. Based on the findings of the pretest, the required modifications were done. Those girls participating in the pilot study were excluded from the study.

Statistical analysis

Data entry was done using a compatible personal computer. The statistical analysis was done using SPSS-16 statistical software package and Excel for figures. The content of each tool was analyzed, categorized and then coded. Data were presented using descriptive statistics in the form of frequencies and percentages for qualitative variables. Qualitative studied variables were compared using Chi-square test. Statistical significance was used at P.value $<0.05$. Pearson's Product Moment correlation coefficients were calculated to assess the relationship among the study variables.

\section{Results}

Table (1): Distribution of adolescent girls according to their personal characteristics

\begin{tabular}{|c|c|c|}
\hline & No. $(n=100)$ & $\%$ \\
\hline \multicolumn{3}{|l|}{ Age } \\
\hline$<15$ years & 53 & 53.0 \\
\hline$\geq 15$ years & 47 & 47.0 \\
\hline Mean \pm SD (Range) & \multicolumn{2}{|c|}{$14.25 \pm 1.53(12.0-17.0)$} \\
\hline \multicolumn{3}{|l|}{ Residence } \\
\hline Rural & 30 & 30.0 \\
\hline Urban & 70 & 70.0 \\
\hline \multicolumn{3}{|l|}{ Level of education } \\
\hline Primary & 28 & 28.0 \\
\hline Preparatory & 72 & 72.0 \\
\hline \multicolumn{3}{|l|}{ Birth order } \\
\hline First & 38 & 38.0 \\
\hline Second & 24 & 24.0 \\
\hline Third & 19 & 19.0 \\
\hline Fourth & 13 & 13.0 \\
\hline Fifth & 6 & 6.0 \\
\hline
\end{tabular}

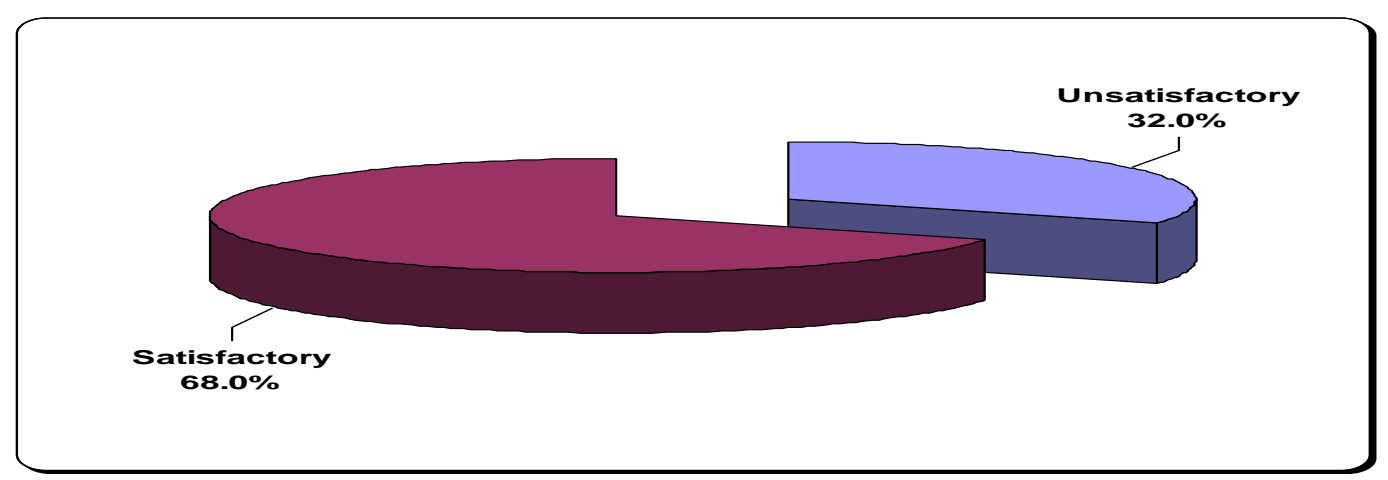

Figure (1): Distribution of participants according to their level of knowledge about menstruation 
Table (2): Relations between level of knowledge and personal characteristics of adolescent girls

\begin{tabular}{|c|c|c|c|c|c|}
\hline & \multicolumn{4}{|c|}{ Level of knowledge } & \multirow[t]{3}{*}{ P-value } \\
\hline & \multicolumn{2}{|c|}{ Unsatisfactory $(n=32)$} & \multicolumn{2}{|c|}{ Satisfactory $(n=68)$} & \\
\hline & No. & $\%$ & No. & $\%$ & \\
\hline \multicolumn{5}{|l|}{ Age } & \multirow[t]{3}{*}{0.680} \\
\hline$<15$ years & 16 & 30.2 & 37 & 69.8 & \\
\hline$\geq 15$ years & 16 & 34.0 & 31 & 66.0 & \\
\hline \multicolumn{5}{|l|}{ Residence } & \multirow[t]{3}{*}{$0.001 *$} \\
\hline Rural & 17 & 56.7 & 13 & 43.3 & \\
\hline Urban & 15 & 21.4 & 55 & 78.6 & \\
\hline \multicolumn{5}{|c|}{ Educational level } & \multirow[t]{3}{*}{$0.000^{*}$} \\
\hline Primary & 18 & 64.3 & 10 & 35.7 & \\
\hline Preparatory & 14 & 19.4 & 58 & 80.6 & \\
\hline \multicolumn{5}{|l|}{ Birth order } & \multirow[t]{3}{*}{0.210} \\
\hline $1-2$ & 15 & 39.5 & 23 & 60.5 & \\
\hline 3 or more & 17 & 27.4 & 45 & 72.6 & \\
\hline
\end{tabular}

$* p<0.05$ (Significant)

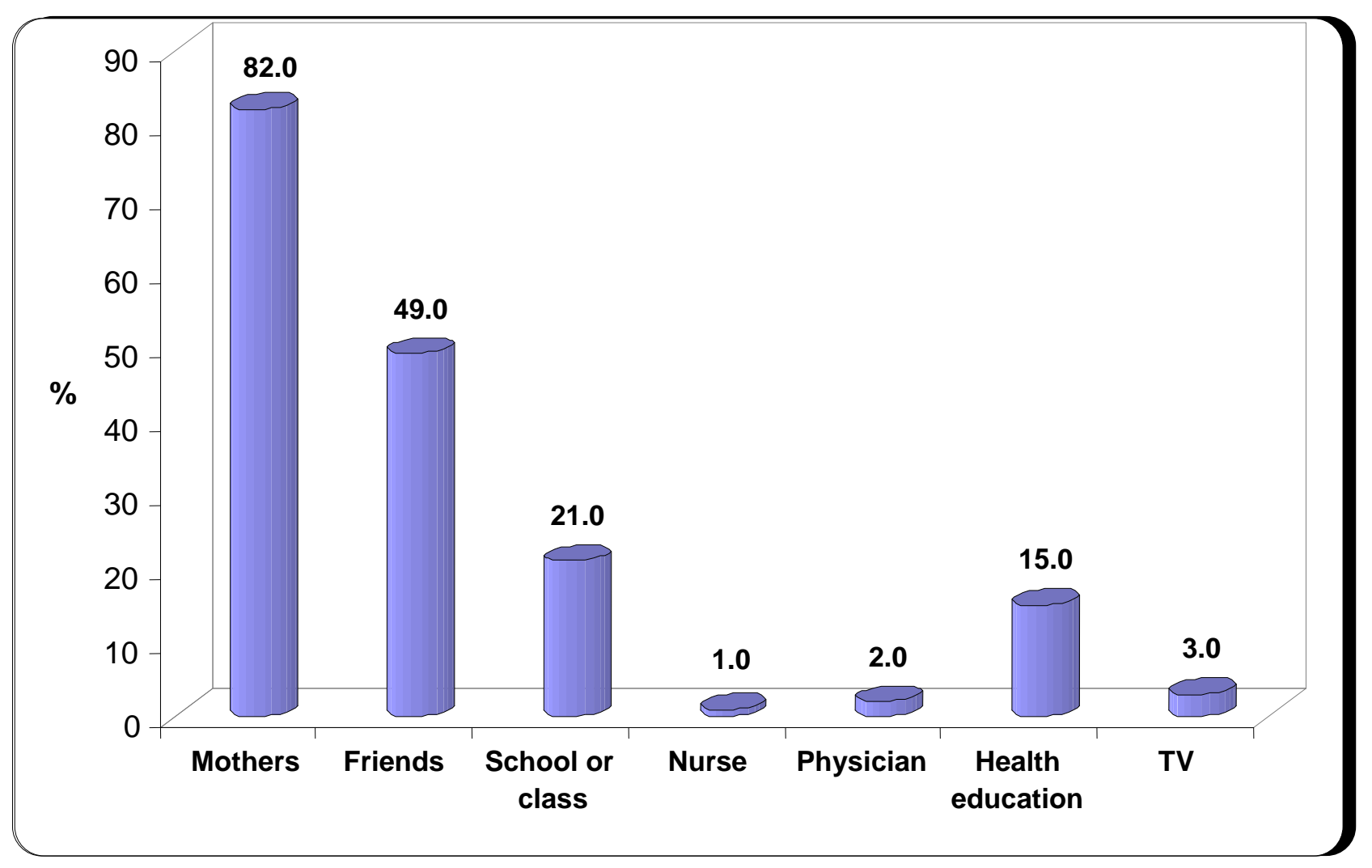

Figure (2): Distribution of adolescent girls regarding source of information about menstruation

More than one answer was found related to participants' sources of information about menstruation. 
Table (3): Distribution of the participants according to their menstrual history

\begin{tabular}{|c|c|c|}
\hline & No. $(n=100)$ & $\%$ \\
\hline \multicolumn{3}{|l|}{ Age at menarche } \\
\hline $9-<12$ years & 12 & 12.0 \\
\hline $12-<15$ years & 74 & 74.0 \\
\hline $15-16$ years & 14 & 14.0 \\
\hline Mean \pm SD & \multicolumn{2}{|c|}{$13.86 \pm 4.71$} \\
\hline \multicolumn{3}{|l|}{ Menstrual regularity } \\
\hline Regular & 82 & 82.0 \\
\hline Irregular & 18 & 18.0 \\
\hline \multicolumn{3}{|l|}{ Days of menstruation } \\
\hline$<5$ days & 65 & 65.0 \\
\hline $5-7 \underline{\text { days }}$ & 33 & 33.0 \\
\hline$>7$ days & 2 & 2.0 \\
\hline \multicolumn{3}{|c|}{ informed about menarche before its onset } \\
\hline Yes & 44 & 44.0 \\
\hline No & 56 & 56.0 \\
\hline \multicolumn{3}{|c|}{ Associated symptoms and signs: ( No=92) } \\
\hline Abdominal pain & 92 & 100.0 \\
\hline Breast tenderness & 16 & 17.4 \\
\hline Tension & 54 & 58.7 \\
\hline Increase weight & 22 & 23.9 \\
\hline Withdrawn and depression & 17 & 18.5 \\
\hline \multicolumn{3}{|l|}{ Having menstrual problem } \\
\hline Yes & 92 & 92.0 \\
\hline No & 8 & 8.0 \\
\hline \multicolumn{3}{|l|}{ Menstrual problem } \\
\hline None & 3 & 3.0 \\
\hline Severe bleeding & 17 & 17.0 \\
\hline Dysmenorrhea & 80 & 80 \\
\hline Tolerable pain & 43 & 43.0 \\
\hline Severe pain & 37 & 37.0 \\
\hline
\end{tabular}

- More than one answer was selected

Table (4) Self-care practices of participants during menstruation

\begin{tabular}{|l|c|c|}
\hline \multicolumn{2}{|l|}{ No. $(\mathbf{n}=100)$} & $\%$ \\
\hline Type of pads used & 5 & 5.0 \\
\hline Piece of old clothes & 10 & 10.0 \\
\hline Piece of new clothes & 85 & 85.0 \\
\hline Sanitary pad & \multicolumn{2}{|c|}{} \\
\hline Methods of drying of pieces of clothes & 57 & 57.0 \\
\hline Expose to the sun & 43 & 43.0 \\
\hline Artificial dry & \multicolumn{2}{|c|}{} \\
\hline Wash clothes of menses & 44 & 44.0 \\
\hline With another of clothes & 56 & 56.0 \\
\hline Wash clothes alone & 21 & 21.0 \\
\hline Methods of disposal of absorbents after use among respondents & 23 & 23.0 \\
\hline Throw it with routine waste & 27 & 27.0 \\
\hline Wash clothes and dry it in the sun & 29 & 29.0 \\
\hline Burn clothes or pad & \multicolumn{2}{|c|}{} \\
\hline Wash clothes and burn pad & \multicolumn{2}{|c|}{} \\
\hline
\end{tabular}




\begin{tabular}{|c|c|c|}
\hline & No. $(n=100)$ & $\%$ \\
\hline \multicolumn{3}{|l|}{ Number of absorbent Pad is changed per day } \\
\hline One & 11 & 11.0 \\
\hline Two & 45 & 45.0 \\
\hline Three & 30 & 30.0 \\
\hline More than Three & 14 & 14.0 \\
\hline \multicolumn{3}{|l|}{ Ideal Thing to Use During Menstruation } \\
\hline Sanitary Pad & 57 & 57.0 \\
\hline Cloth & 19 & 19.0 \\
\hline Both & 24 & 24.0 \\
\hline \multicolumn{3}{|l|}{ Absorbents in Practice among the Respondents } \\
\hline Sanitary Pad & 55 & 55.0 \\
\hline Cloth & 21 & 21.0 \\
\hline Both & 24 & 24.0 \\
\hline \multicolumn{3}{|l|}{ Taking bath in all days of menstruation } \\
\hline Yes & 89 & 89.0 \\
\hline No & 11 & 11.0 \\
\hline \multicolumn{3}{|c|}{ Healthcare to subside premenstrual signs and symptoms } \\
\hline Physical rest & 7 & 7.0 \\
\hline Analgesic & 75 & 75.0 \\
\hline Warm bath & 36 & 36.0 \\
\hline Exercise & 19 & 19.0 \\
\hline Warm fluids & 23 & 23.0 \\
\hline \multicolumn{3}{|l|}{ Health care to subside dysmenorrhea } \\
\hline Consulting a physician & 12 & 12.0 \\
\hline Taking over the counter drugs or self-medication & 65 & 65.0 \\
\hline Warm bath & 35 & 35.0 \\
\hline Analgesic & 32 & 32.0 \\
\hline Physical rest & 10 & 10.0 \\
\hline \multicolumn{3}{|l|}{ Exercise during menstruation } \\
\hline Yes & 69 & 69.0 \\
\hline No & 31 & 31.0 \\
\hline \multicolumn{3}{|l|}{ The dietary or nutritional influence on menstruation } \\
\hline Yes & 50 & 50.0 \\
\hline No & 50 & 50.0 \\
\hline \multicolumn{3}{|l|}{ Absenteeism from school in the first day } \\
\hline Yes & 15 & 15.0 \\
\hline No & 85 & 85.0 \\
\hline
\end{tabular}

- More than one answer was selected

Table (5): Distribution of the participants according to their scores of attitudes toward menstruation

\begin{tabular}{|l|c|c|c|c|}
\hline Response toward menstruation & No. $(\%)$ & Score & Mean \pm SD & Range \\
\hline It is debilitating & $42(42)$ & 60 & $40.64 \pm 6.62$ & $29.0-58.0$ \\
\hline Is bothersome & $15(15)$ & 30 & $21.22 \pm 3.79$ & $14.0-30.0$ \\
\hline It is natural & $53(53)$ & 20 & $14.59 \pm 3.37$ & $5.0-20.0$ \\
\hline They can anticipate it & $80(80)$ & 20 & $13.78 \pm 2.89$ & $6.0-20.0$ \\
\hline They denied any effect of it & $8(8)$ & 35 & $23.90 \pm 4.97$ & $13.0-35.0$ \\
\hline Total attitude & & $\mathbf{1 6 5}$ & $\mathbf{1 1 4 . 1 3} \pm \mathbf{1 7 . 4 0}$ & $\mathbf{8 1 . 0}-\mathbf{1 5 7 . 0}$ \\
\hline
\end{tabular}




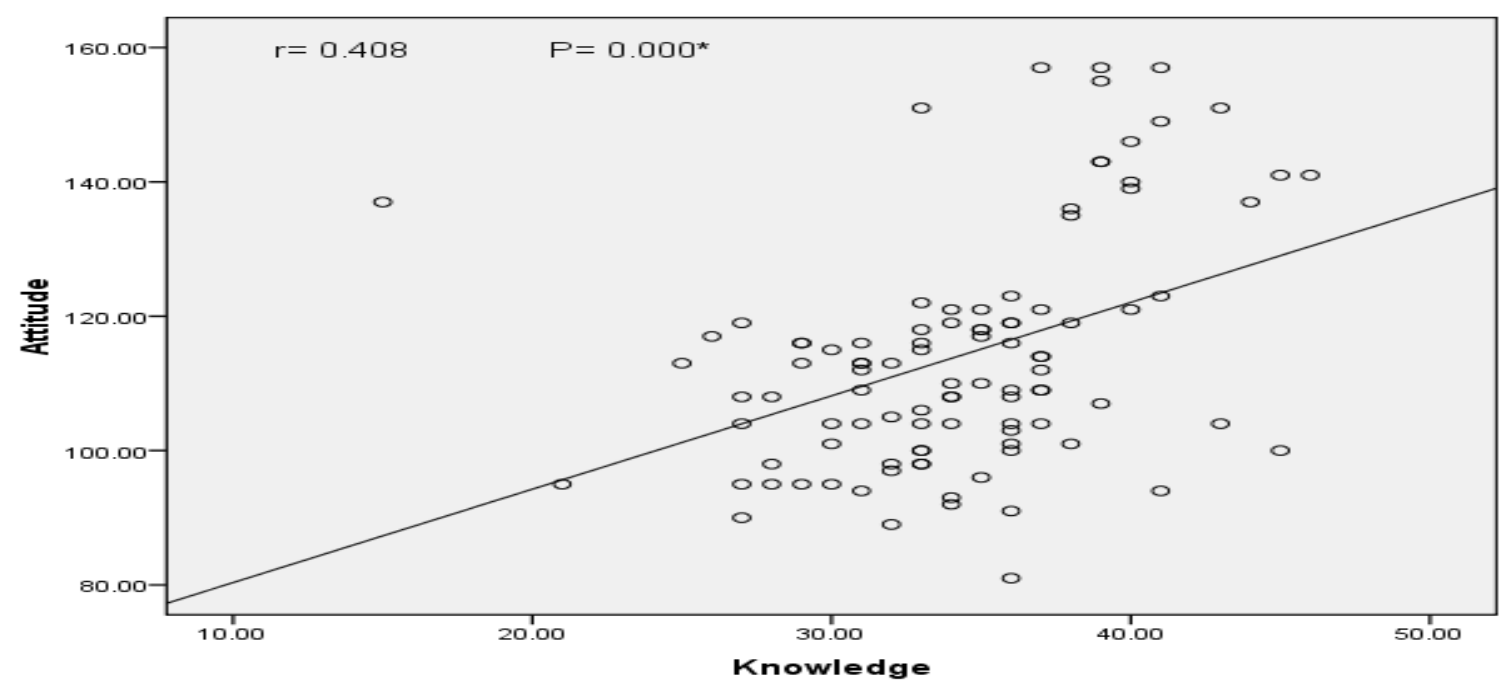

Figure (3): Correlation between knowledge and attitudes among participants

Table (1): Showed personal characteristics of the participating girls. .More than half of girls were $<15$ years $(53 \%)$, where the mean age of them was 14.25 \pm 1.53 years and the majority of them were urban (70\%)and in the preparatory level. As regards birth order, $38 \%$ of adolescent girls were the $1^{\text {st }}$ girl in the family, $6 \%$ of them were the $5^{\text {th }}$.

Table (2): Showed the relation between the level of knowledge and personal characteristics of adolescent girls. The level of knowledge was more significantly higher among urban girls and those with a higher level of education $(\mathrm{P}=0.001,0.000$ respectively. Otherwise, no relation either between the level of knowledge and age or birth order of adolescent girls.

Figure (1): As regard to participants' knowledge about menstruation, this study indicated that $68 \%$ of participants had satisfactory while $32 \%$ had unsatisfactory levels knowledge.

Figure (2): Clarifies the source of information about menstruation for participant adolescent girls. The majority of girls $(82 \%)$ said that, their mothers were the main source of information followed by friends $(49 \%)$, school or class $(21 \%)$, health education in society $(15 \%)$, TV (3\%), physician $(2 \%)$, and nurse $(1.0 \%)$.

Table (3): As regard menstrual history, the mean age of menarche of the respondents was $13.86 \pm 4.71$ years. Eighty-two percent of the adolescent girls stated that they have a regular menstrual cycle. The duration of menstruation was $<5$ days among $65 \%, 5$ 7 days among 33\%and $>7$ days among $2 \%$ of an adolescent girl. In addition, $44 \%$ of participant adolescent girls had been informed about menarche before its onset. The majority of participants, $(92.0 \%)$ suffered from various types of menstrual problems.
Dysmenorrhea represented $80 \%$ including tolerable pain $(43 \%)$ and severe pain $(37 \%)$ followed by severe bleeding (17\%).

Table (4): As regard self-care practice during menstruation, $85 \%$ of adolescent girls are using a sanitary pad, $10 \%$ of them using pieces of new clothes and $5 \%$ of them using pieces of old clothes. It was found that $57 \%$ of the girls dried their washed reusable absorbent materials (cloths) by exposing it to the sun; while $56 \%$ of them are washing their clothes of menstruation alone. For final disposal of the menstrual pad, 29\% of the girls are washing clothes and burning pad followed by $27 \%$ are burning clothes or pad, $23 \%$ are washing clothes and drying them in the sun and $21 \%$ are throwing them with other wastes. In addition $45 \%$ of girls are changing their pads two times a day. More than two-thirds (67\%) of participants reported that sanitary pad is the ideal thing to use during menstruation while $24 \%$ of them are using both sanitary pads and cloths. The majority of participants $(89 \%)$ reported that they are practicing the personal health taking behavior, such as taking a bath in all days of menstruation. The majority of participants $(75 \%$,) reported that the subsidence of premenstrual symptoms and signs by analgesic, $36 \%$ by a warm bath and $23 \%$ by warm fluids. Also, $65 \%$, of participants, are using the counter drugs or self-medication and $12 \%$ of them are consulting a physician. Sixty-nine percent of the adolescent girls reported that they don't practice any kind of exercise during menstruation. Also, half of adolescent girls believe that some diets or nutritional interventions can influence the menstruation. In this study, $15 \%$ participants stated that they are absent from school during menstruation. 
Table (5): illustrated distribution of the participants according to their scores of attitudes toward menstruation. The participants agreed that, menstruation is a debilitating (42\%) $(40.64 \pm 6.62)$, a bothersome (15\%) $(21.22 \pm 3.79)$ and natural event $(53 \%)(14.59 \pm 3.37)$. Furthermore, they agreed that they can anticipate their menstruation $(80 \%)(13.78$ $\pm 2.89)$ and they denied the effect of it $(8 \%)(23.90 \pm$ 4.97).

\section{Discussion}

The onset of menstruation is one of the most important changes occurring among girls during the adolescent years (Dhingra, et al., 2009 \& Diaz et al, 2006). Handling menstruation is considered a major challenge for every adolescent girl which is a normal body function in females (Begum et al., 2009). The aim of the current study was to assess the knowledge, attitude and practice toward menstruation among adolescent girls. The current study showed that urban adolescent girls had a more satisfactory level of knowledge than girls living in the rural setting. As shown in the table (1). These findings are similar to a study of George, (2012) and those conducted by Jarrah \& Kamel(2012).This could be explained by the fact that, a high proportion of urban residents could have accessible reproductive health care service and better decision-making autonomy than rural female students. In addition, this difference might also be attributable to the difference in implementation of relevant health intervention programs (Joshi, 2006).

This study indicated that more than two-thirds (68\%) of participants had a satisfactory level of knowledge related to menstruation as shown in figure (1). This finding is agreed with that of Lawan et al., (2010) who stated that girls had a fair knowledge about menstruation. Similarly, Singh, (2006); Ali and Rizvi, (2010), \& Prateek \& Saurabh, 2011)reported that the girls had good knowledge about menstruation. In addition, Shanbhag et al, (2012) demonstrated that knowledge of menstruation among female high school students was high. This might be attributed to the time gap that accessibility, availability and ability to optimally utilize reproductive health information may be improved as time increases. However, in India Thakre et al., (2010) said that among the adolescent girls in both urban and rural areas, the knowledge on menstruation is poor and the practices are often not optimal for proper hygiene.

In this study, $44 \%$ of participants had been informed about menarche before its onset as shown in the table (3). This finding is supported by the study conducted by Eswi et al., (2012) who reported that $74 \%$ of the participants had been informed about menarche before its onset. Similarly, Myoung \& Young, (2013) mentioned that $67 \%$ girls were well prepared and had knowledge about menstruation.

In the current study, $82 \%$ of adolescent girls mentioned that, their mothers were the main source of information as shown in figure 2, This could be due to lack of proper communication between mother and daughter owing to traditional taboos, they feel awkward and embarrassed to discuss on this subject. These results are in agreement with the results of Shanbhag et al., (2012), Sapkota et al.,(2013), Khudair, (2013) \& Sherestha et al., (2013) studies who found that majority of girls were informed by mothers. Similar results were reported by United Nations, educational, scientific \& cultural organization, (2014). This brings out an important issue of the feasibility of involving parents in educating their children regarding reproductive health problems especially mother as young girls usually propound her emotional and psychological problems with her mother. This does not augur well for the future mothers who are expected to have a good practice of menstruation and are expected to carry the message to their young ones. In contrast, with this study Interagency youth working group, (2007) found that the primary sources of information about menstruation for girls were school teachers. Thus, it is important to ensure that school teachers who deliver information and education on sexuality receive adequate training and ongoing support and supervision. Evidence showed that when teachers receive training and support in their role as sexuality educators they feel more comfortable and equipped to provide effective, objective and nonjudgmental sexuality education. In addition, it should be ensured that a variety of context-specific teaching methods is used in providing sexuality education to young people.

On the other hand, Juyal et al.,(2012) reported that friends were the first source of information about menstruation for $31.8 \%$ of adolescent girls in India. Also, Bayray, (2012) reported that friends were considered the main contributors for getting the knowledge $(50 \%)$ followed by mothers $(36 \%)$ and aunts/relatives (19\%) (Nagar andAimol, 2010). A study was conducted In Mansoura -Egypt, by ElGilany et al., (2005), they found that $92.2 \%$ of the girls reported mass media as their main source of information about menstruation followed by mothers $(45 \%)$.

As regard menstrual history, the mean age of menarche of the respondents was found to be $13.86 \pm$ 4.71 years as shown in table 3 , this result is similar to finding that of Abdel-Hameed et al., (2011) and Yassin, (2012) in Egypt. Different ranges of age of 
menarche by different studies in different countries as El -Gilany et al, (2005) among Egyptian female adolescents found Mean \pm SD was $(12.87 \pm 1.29$ years) and Nemade et al., (2009) stated that mean age of menarche among Jordanian girls was (13.8 years).This difference could be attributed to the influence of both heredity and socioeconomic conditions, mainly nutrition. Girls from upper socioeconomic class have reached menarche at an earlier age than those from the lower socio-economic class as they attain a certain critical weight at an early age due to improved nutrition and better health (Ali et al., 2011).

Regarding menstrual problems, in the current study, the participants reported that dysmenorrhea $(80 \%)$ including tolerable pain $(43 \%)$ and severe pain $(37 \%)$ was the major problem followed by severe bleeding (17\%).as shown in table 3 These results are agreeing with the results of several studies in different parts of the world that, dysmenorrhea was the most common medical problem among the girls during menstruation (Dasgupta, \& Sarkar, 2008, Omidvar \& Begum, 2010, Sapkota et al., 2013 \& Reda et al., 2014).

As regard self-care practice during menstruation among adolescent girls in this study, more than half $57 \%$ of the girls reported that they are drying their washed reusable absorbent materials (cloths) by exposing it to the sun, $56 \%$ of them reported that they are washing their clothes of menstruation alone while $29 \%$ of them washing and burning the used cloth. Despite the majority of the participants reported that sanitary pad was the ideal thing to use during menstruation, only $55 \%$ of participants actually used it. As shown in table 4. These findings are supported by El-Lassy and Madian, (2013) who observed a poor perception and behavior towards the use of sanitary pads during menstruation among adolescents girls. They noted that lack of money was responsible for non-usage of sanitary pads and hence the use of another absorbent such as clothes. Similar results were reported by other studies carried out among the same population in developing countries (Palep \& Singh, 2007). Therefore undoubtedly poverty and poor financial resource play a major role on the choices of absorbents leading to the use of unsanitary materials.

In the current study the Majority of girls $89 \%$ reported that they are practicing the personal health taking behavior, such as taking a bath in all days of menstruation as shown in table 4, this finding is consistent with those of Thakre et al., (2010) \& Gaudineau, et al., (2010) who reported that, $11 \%$ of participants reported not taking a daily bath during menstruation.

Regarding the use of analgesia, $75 \%$ of participants reported subsidence of premenstrual symptoms by analgesic, $36 \%$ are using warm bath while $23 \%$ of them are drinking warm fluids to subside the premenstrual signs and symptoms, as shown in table 4. These findings are similar to that of Al-Asadi \& Abdul-Qadir (2013) who found that $56.4 \%$ of the girls used analgesia without prescription to relieve menstrual cycle pain in Iraq. Such a high percentage is of major concern, endangering adolescent girls for overuse and side effects. On the other hand, only $22.4 \%$ of adolescent girls in Haryana, India used analgesics without prescription during menstruation (Dasgupta \& Sarkar, 2008)

In the present study nearly two-thirds $(65 \%$, ) of the participants reported that they are taking over the counter drugs or self-medication while $12 \%$ of them are consulting a physician to subside dysmenorrheal As shown in table 4. This finding is similar to the result of Jasrotia et al., (2009) study who reported that $67 \%$ of students with dysmenorrhea selfmedicated with over-the-counter drug preparations and only $18 \%$ consulted a physician for these problems. Also ,Al-Asadi \& Abdul-Qadir (2013), reported that $13.7 \%$ of adolescent girls consulted a physician to subside dysmenorrhea and added that, this suboptimal use of the health care system or low consultation rate by adolescents has been reported in Egypt by Mohamed (2012). This may be attributed to socio-cultural factors. Cultural background and the fact that most women regard menstruation as a normal part of their female constitution and a private and embarrassing issue to be discussed publicly may explain such behavior. Therefore, educating young females to consult a doctor for their menstrual problems, will not only cause the more effective pain relief but also prevent them from the possibility of adverse drug reactions.

The current study revealed that $69 \%$ of the adolescent girls reported that, they didn't practice any kind of exercise during the menstrual cycle as shown in table 4 which is in agreement with the results of Jasrotia et al.,(2009) who stated that, majority of participants $(89 \%)$ didn't practice any kind of exercise during the menstrual cycle. They explained this behavior by the fear that participation in strenuous exercise might harm their reproductive organs, and she cannot compete successfully in sports because their menstrual blood might "stain" the playing fields.

In the current study, half of adolescent girls believe that some diets or nutritional interventions can influence the menstruation. This result was not in agreement with Jasrotia et al., (2009) who reported that only $8 \%$ of subjects believe that, some diets or nutritional interventions can influence such as fruits, vegetables, and corns, along with avoiding too much salt and sugar. This could be attributed to changing 
trends in lifestyle, dietary habit or some medical reasons.

In this study, $15 \%$ of adolescent girls reported an absence from school during menstruation, this results is supported by the study of Esimai \& Esan, (2010) who observed that menstruation was the leading cause of absenteeism of women from work, school, and other activities. This may be related to fears about bleeding in front of her colleagues in the class or work.

In the present study, there was a positive correlation between participants' score of knowledge and their attitude toward menstruation as shown in figure 3 . This finding is in agreement with the results Reda et al., (2014) who noted that, participants' score of knowledge positively correlated with their attitude toward menstruation.

The effect of obtaining proper knowledge about menstruation on the girls' attitude was proved by several researchers (Hassanen et al., 2004) where they found that girls who were adequately prepared have more positive experiences with menstruation. The result of the present study highlighted the same idea as it was found that participants' score of knowledge was positively correlated with their attitude toward menstruation. Good preparation for menstruation coupled with positive menarche experience would be related to more positive adult menstrual attitudes, experiences, and behaviors.

This highlights that our society is still bounded by social, cultural taboos which lead to the negative attitude of adolescents towards menstruation. This demonstrates the urgency of the situation that needs effective interventions to address the restrictions which are imposed or practiced by adolescent girls.

\section{Conclusions and Recommendations}

According to the study findings and its discussion, the present study can conclude that adolescent girls had a satisfactory level of knowledge about menstruation. Their mothers were the main source of information. The level of knowledge positively correlated with the girls' attitude toward menstruation It is recommended to prepare girls for menstruation before menarche through providing them with wellplanned school health program for girls and mothers about menstruation by well-trained educators. Parents should be made to acknowledge the need to support their children at school with sanitary menstrual absorbents in addition to other basic hygienic products.

\section{References}

1. Abdel-Hameed N., Maher S., Mohamed, Nadia H., Ahmed \& Eman R., Ahmed.,
(2011): Assessment of Dysmenorrhea and Menstrual Hygiene Practices among Adolescent Girls in Some Nursing Schools at EL-Minia City, Egypt. Journal of American Science; 7(9):216-223.

2. Eswi A., Helal H., Elarousy W., (2012): Menstrual Attitude and Knowledge among Egyptian Female Adolescents, Journal of American Science. 8(6):555-565.

3. Adinma E., (2008): Perceptions and practices on menstruation amongst Nigerian secondary adolescent girls. Afr. Repro. Health, 12 (1):7483.

4. Ali, A., Rayis D., Mamoun M., Adam I., (2011): Age at menarche and menstrual cycle pattern among schoolgirls in Kassala in eastern Sudan. 3(March):111-4.

5. Ali, S., \& Rizvi, N., (2010): Menstrual knowledge and practices of female adolescents in urban Karachi, Pakistan. J. Adolesc. 33(4):531-541.

6. Al-Asadi J., \& Abdul-Qadir R., (2013). Dysmenorrhea and its impact on daily activities among secondary school students in Basra, Iraq. J Fac Med Baghdad: 55(4): 339-344.

7. Anjum F., Zehra N., Haider G., Rani S., Siddique A., Munir A., (2010): Attitudes towards menstruation among young women. Pak J Med Sci; 26(3):619-622

8. Begum J., Hossain A., Nazneen S., (2009): Menstrual pattern and common menstrual disorders among students in Dinajpur medical college. Dinajpur Med Col J.; 2 (2):37-43.

9. Bayray A., (2012): Menstrual perceptions and preparation of rural adolescent females in Tigray, North Ethiopia.Universal Journal of Education and General Studies 1(1): 009-016.

10. Firat M., Kulakaç O., Oncel S., Akcan A., (2009). Menstrual Attitude Questionnaire: confirmatory and exploratory factor analysis with Turkish samples. J Adv Nurs. 2009 Mar;65(3):652-62.

11. Çakmakci E., Sanioğlu A., Patlar S., Çakmakçi O., Çinar V., (2005): Effect of Menstruation on Anaerobic Strength, SPORMETRE BedenEğitimiveSpor. BilimleriDergisi, III (4) 145-149

12. Çevirme A., Çevirme H., Karaoğlu L., Uğurlu N., korkmaz Y., (2010): The Perception of Menarche and Menstruation Among Turkish Married Women: Attitudes, Experiences and Behaviours, Social Behavior and Personality, 38(3),381-394.

13. Dasgupta A., \& Sarkar M., (2008): Menstrual Hygiene. Indian J Community Med. 33(2):77-80. 
14. Dhingra R., Kumar A., Kour M., (2009): Knowledge and practices related to menstruation among tribal adolescent girls. Ethno-Med. 3(1): 43-48.

15. Diaz A., Laufer M., and Breech L., (2006): Menstruation in girls and adolescents: using the menstrual cycle as a vital sign. Pediatrics 118: 2245-2250.

16. El-Gilany A., Badawi K., El- Fedawy S., (2005): Menstrual hygiene among adolescent girls in Mansoura, Egypt. Reproductive Health Matters.13 (26): 147-152.

17. El-Lassy B., \& Madian A., (2013): Impact of Health Education Program on Menstrual Beliefs and Practices of Adolescent Egyptian Girls at Secondary Technical Nursing School. Life Sci.; 10(2):335-345.

18. Esimai O., Esan O., (2010): Awareness of menstrual abnormality amongst college students in the urban area of ile-life, osun state, Nigeria. Indian Journal Community. Med 35: 63-66.

19. Fetohy EM. (2007): The impact of a Health Education Program for Secondary School Saudi Girls About Menstruation at Riyadh City. J Egypt Public Health Assoc 82 (1\& 2),

20. Gaudineau N., Hawai S., Altuwaijri S., (2010): Prevalence and pattern of menstrual disorders among Lebanese nursing students. Eastern Mediterranean Health Journal EMHJ. 18(4) 346352.

21. George C., (2012): Health of the world's adolescents: a synthesis of internationally comparable data. Lancet 379: 1665-75: 10.

22. Hassanen R., Tosson M., Abd El-Elkareem H., (2007): Effect of health education and training program for secondary school students on menstruation at Assiut City. Bulletin of High Institute of Public Health. 2007; 34(3): 575-94.

23. Houston A., Abraham A., Huang Z., D'Angelo L., (2006): Knowledge, attitudes, and consequences of menstrual health in urban adolescent females. J PediatrAdolesc Gynecol. 19:271-5.

24. Interagency youth working group (2007): Helping parents improve adolescent health. Family health international. USA.

25. Jarrah S. \& Kamel A.., (2012): Attitudes and practices of school-aged girls towards menstruation. International Journal of Nursing Practice 2012; $18: 308-315$

26. Jasrotia R, Kanchan A, Hathi G, Harsoda J., (2009): Knowledge, attitude and practices of Indian girls on various aspects of menstruation. Transworld Medical Journal.1(1):14-7.

27. Joshi B., (2006): Reproductive and health problems and health seeking behavior among the adolescents in urban India. Indian Journal of Pediatrics; 73:509-13.

28. Juyal R., Kandpal S., Semwal J., Negi K., (2012): Practices of menstrual hygiene among adolescent girls in a district of uttarakhand. Indan Journal Community Health. 24(2):124-8.

29. Khudair F., (2013): Assessment the Level of awareness of Female Students toward menstruation in Nursing College Kufa Journal for Nursing. 3 (1): 57-62.

30. Lawan U., Nafisa Y., \& Aisha M., (2010): Menstruation and menstrual hygiene amongst adolescent girls in Kano, Northwestern Nigeria. African Journal of Reproductive Health 14(3): 201-207.

31. LiPing W., \& MingK E., (2011): Menstrualrelated attitudes and symptoms among multiracial Asian adolescent females. International Journal of Behavioral Medicine. 18(3): 246-253.

32. Marván M., Cortés-Iniestra S., Gonzáles R., (2005): Beliefs about and attitudes toward menstruation among young and middle-aged Mexicans.53:273-9.

33. Mohamed E., (2012): Epidemiology of dysmenorrhea among adolescent students in Assiut city, Egypt. Life Science Journal; 9(1): 348-353.

34. Moloud F., Zeinab H., Nayereh A., Hajikhani G., \& Abdulhay K., (2012): Promoting menstrual health among Persian adolescent girls from low socioeconomic backgrounds: a quasiexperimental, 38(3), 381-394.

35. Myoung K. \& Young Y., (2013): Knowledge of Menstruation, Emotional reaction to menarche, Attitude toward menstruation and coping behavior among Korean primary school students Korean J Women Health Nurs.15 (1): 64-72.

36. Nagar S., \& Aimol R., (2010): Knowledge of Adolescent Girls regarding menstruation in tribal Areas of Meghalaya Stud Tribes, 8: 27-30.

37. Nemade I., Anjenaya S., Gujar R., (2009): Impact of health education on the knowledge and practices about menstruation among the adolescent girls of Kalamboli, Navi Mumbai. Health and Population: Perspective Issues. 32(4):67-75.

38. Nese Z., (2013): Evaluation of Menstrual Attitude of Collegiate Athletes, Life Science Journal 2013; 10(6s):295-300.

39. Omidvar S., \& Begum K., (2010): Factors influencing hygienic practices during menses among girls from south India- A cross-sectional study Factors influencing hygienic practices during menses among girls from south India- A cross-sectional study. Int $\mathbf{J}$ Collaborative Res Intern Med Pub Health. 2(12):411-23. 
40. Palep F., \& Singh M., (2007): Epidemiology of abnormal uterine bleeding. Best Practice \& Research. Clinical Obstetrics \&Gynaecology, 21:887-890.

41. Prateek B., \& Saurabh S., (2011): A crosssectional study of knowledge and practices about reproductive health among female adolescents in An Urban Slum of Mumbai. Journal of Family and Reproductive Health 5(4): 117-124.

42. Jasrotia RB, Kanchan A, Hathi G K and Harsoda JM. (2009): Knowledge, attitude and practices of Indian girls on various aspects of menstruation. Transworld Medical Journal. 1(2):3741

43. Reda I., Maha M., Hanan H., (2014): Effect of Health Education Program on Knowledge and Practices about Menstrual Hygiene among Adolescents Girls at Orphanage Home.IOSR Journal of Nursing and Health Science; 3, (6) : 48-55.

44. Sadiq M., \& Salih A., (2013): Knowledge and Practice of Adolescent Females about Menstruation in Baghdad. J Gen Pract 2: 138.

45. Sapkota D., Sharma D., Budhathoki S., Khanal V., Pokharel H., (2013): Knowledge and practices regarding menstruation among school going adolescents of rural Nepal, Journal of Kathmandu Medical College, Vol. 2, No. 3, Issue 5, Jul.-Sep.

46. Shanbhag D., Shilpa R., Souza N., Josephine P., Singh J., et al., (2012): Perceptions regarding menstruation and practices during menstrual cycles among high school going adolescent girls in resource-limited settings around Bangalore city, Karnataka, India. International Journal of Collaborative Research on Internal Medicine \& Public Health 4(7): 1353-1362.

47. Sherestha S., Jaita M., Rajaina T., (2013): Knowledge attitude and practices towards menstrual hygiene among adolescent girls. International Journal of nursing education, 5 (11), 221-27.

48. Shokry E., Allah A., Elsayed E., Elsabagh M., (2012): Impact of Health Education Intervention on Knowledge and practice about Menstruation among Female Secondary School Students in Zagazig City. J Am Sci. 7(9):737-47.

49. Singh A., (2006): Place of menstruation in the reproductive lives of women in rural North India. Indian J Community Med. 31(1):10-4.

50. Sudenshana R., Dasgupta A., (2012): Determinants of menstrual hygiene among adolescent girls: A multivariate analysis. Natl J Community Med. 3(2):294-301.
51. Thakre SB., Thakre SS., Reddy M., Rathi N., Pathak K., Ughade S. (2010): menstrual hygiene knowledge and practice among adolescent girls of saoner, Nagpur District. J ClinDiag Res. 5(5):1027-33.

52. United Nations, educational, scientific \& cultural organization (UNESCO) (2014): Puberty education and menstrual hygiene management. Available: http://unesdoc.unesco.org/images/0022/00 2267/226792e.pdf-puberty. Accessed 2014 April 25.

53. Wong L., \& Khoo E., (2011): Dysmenorrhea in a multiethnic population of adolescent Asian girls.Int J Gynaecol Obstet. 108(2):139-42.

54. Yassin SA. (2012): Herbal Remedy used by Rural Adolescent girls with Menstrual Disorders. Journal of American Science; 8(1):467-473 